\title{
LA IDENTIDAD URBANA COMO CATEGORÍA DE ANÁLISIS. Una propuesta metodológica para la lectura del territorio a través de la consolidación Histórico-Espacial de sus atributos urbanos característicos.
}

\author{
Ana Cristina Herrera Valencia \\ Universidad Pontificia Bolivariana (Medellín) \\ Evelyn Patiño Zuluaga \\ anacristinaherreravalencia@gmail.com
}

\section{RESUMEN}

Reconocer la ciudad como una construcción en tiempo y espacio, es clave para una lectura territorial integral, que al combinar diferentes factores, desde la dimensión natural, social e histórico-urbana, permite identificar las condiciones existentes en el territorio, que marcan el punto de partida para sus oportunidades futuras. La lectura territorial a la luz de la identidad, se propone a partir de un marco de criterios de análisis por medio de los cuales, se reconocen las particularidades que identifican a cada asentamiento, configurando sus atributos urbanos identitarios. En el siguiente artículo se realiza la descripción de la propuesta metodológica que se configura como un sistema fijo aplicable en diferentes escalas y contextos.

Palabras Clave: Identidad Urbana, Lectura Territorial, Atributos urbanos.

\begin{abstract}
Recognizing the city as a construction in time and space is key to make a comprehensive territorial reading, that combining different factors from the natural, social and historic urban dimensions will lead to identify the existing conditions in territory, those who mark the starting point for future opportunities. Territorial comprehension from an identity point of view is proposed from a framework of analysis criteria, by which the peculiarities that identify each settlement are recognized, shaping their urban identity attributes. The following paper describes the methodological proposal, that is set up as a fixed system applicable in different scales and contexts.
\end{abstract}

Key Words: Urban identity, territorial reading, urban attributes. 


\section{INTRODUCCIÓN}

Las identidades son construcciones que se dan mediante un proceso de individualización, una construcción que implica la interrelación de diversos factores propios de un contexto (Castells, 1998). Estos factores, están asociados con actividades y relaciones que prescriben las formas de vida de una zona geográfica determinada (Harner, 2001). Es así que la identidad, considera un proceso continuo a través del tiempo, en el cual las sociedades procesan los elementos que ofrece el contexto y los reordenan, imprimiéndoles características particulares, "según las determinaciones sociales y los proyectos Culturales implantados en su estructura social y en su marco espacial/temporal" (Castells, 1998: 29).

En lo referente a la Identidad, existen diferentes autores que han relacionado este concepto con el de Desarrollo, reconociendo la necesidad de valorar y conocer las posibilidades de aprovechamiento del suelo, de los recursos y del capital humano, como claves para la configuración de un sistema sólido y competitivo; Alberto Magnaghi, Saskia Sassen, John Harner, Zorán Roca, entre otros. Las diferentes iniciativas por abordar el concepto de la identidad en relación con el territorio, se orientan a reconocer la influencia del espacio en la construcción de la identidad territorial; es decir, ponen en manifiesto una relación indivisible entre la sociedad y su medio físico de localización, evidenciando el dominio que tiene el espacio en las diferentes expresiones sociales, culturales y económicas. Sin embargo, las características urbanas físico-espaciales particulares de cada territorio, no son estudiadas a fondo como aspectos claves propios de la identidad territorial, en los cuales pueden leerse y evidenciarse diferentes aspectos de la cultura y las economías locales.

Lo anterior, supone una necesidad de asociar el concepto de identidad urbana, a una estrategia metodológica que posibilite una lectura integral del territorio, a partir del reconocimiento de las transformaciones urbanas a lo largo del tiempo, que conforman los atributos espaciales característicos asociados a un territorio y sus valores identitarios. Para abordar esta temática, se propone en un primer momento la aproximación al concepto de la identidad urbana, para luego establecer los criterios propuestos como base de la metodología de lectura territorial.

\section{LA IDENTIDAD URBANA, CONCEPTUALIZACIÓN Y METODOLOGÍA DE ANÁLISIS}

\subsection{El concepto de Identidad, posturas y perspectivas}

La palabra Identidad, es definida por el diccionario a través de diversas acepciones, entre las que se pueden resaltar dos para efectos de la relación social con el espacio y el tiempo. La primera, propone a la identidad como un conjunto de rasgos propios de un individuo o de una colectividad que los caracterizan frente a los demás; y la otra por su parte, la relaciona con la conciencia que una persona tiene de ser ella misma y distinta a las demás. ${ }^{1}$ Para estos dos casos, no se hace referencia al territorio ni al espacio físico, no obstante, es un punto de partida desde el cual se entiende que la identidad requiere necesariamente el reconocimiento de un conjunto de características que permiten la diferenciación de un individuo o una colectividad, respecto a un conjunto general. La identidad es un concepto complejo y heterogéneo, que reúne diferentes factores propios del individuo, la sociedad y su medio de desarrollo. Por lo anterior, es una temática susceptible de ser estudiada desde diversos campos analíticos, que remiten a los distintos niveles de la acción social (Portal, 1991) y a los múltiples componentes a partir de los cuales se puede explicar la construcción subjetiva del individuo y la construcción colectiva de la comunidad. La construcción de identidad colectiva, implica necesariamente la relación con otras sociedades y culturas, teniendo en cuenta que debemos considerar las identidades en términos de experiencias y relaciones y que solo a través de los contrastes y diferencias, es posible reconocer características identitarias. (Hall, S; Du Gay P, 2011)

Una de las principales características asociadas al concepto de identidad, tiene que ver con el carácter colectivo de su construcción y con las transiciones temporales en las que se enmarcan sus referentes simbólicos; en este sentido, Stuart Hall hace especial hincapié, en que las identidades nunca se unifican Están cada vez más fragmentadas y fracturadas; Nunca son singulares, sino construidas de múltiples maneras a través de discursos prácticas y posiciones diferentes. (Hall, S. Du Gay, P, 2011:17) Por este carácter de construcción permanente, la Identidad se ha asociado comúnmente con un pasado histórico con el cual se establecen relaciones constantes

\footnotetext{
${ }^{1}$ Definiciones de la Real Academia Española
} 
de correspondencia, teniendo que ver con cuestiones referidas al uso de los recursos de la historia, la lengua y la cultura; en este orden de ideas, Hall propone que el concepto de identidad no se limita a "quienes somos" o "de dónde venimos", sino en qué podríamos convertirnos.

La identidad como concepto ha sido tratada desde diferentes puntos de vista, con especial énfasis por las ciencias sociales como la antropología, la psicología y la sociología, también desde la filosofía e incluso desde la concepción ecológica del territorio y sus características. Zygmount Bauman, sitúa el origen del concepto de la identidad en la modernidad; en los tiempos modernos se identificó la necesidad de "construir" la identidad, a través de referentes que perduraran en el tiempo con los cuales se propiciara una cohesión social; lo anterior, obedece a un ambiente de descontextualización de los diferentes factores propios de la sociedad y la cultura, los cuales se vieron alterados por un flujo en la comunicación que interrelacionó diferentes territorios y culturas, desdibujando límites y compartiendo experiencias, bajo las cuales era necesaria la configuración individual de la identidad colectiva. (Bauman en Castells, 1998:41)

\subsection{La Identidad desde el urbanismo}

Una de las razones fundamentales para considerar la importancia de la identidad territorial desde el punto de vista físico, radica en la relevancia de las ciudades para la sociedad contemporánea, teniendo en cuenta que en los ámbitos urbanos se asienta la mayor parte de la población mundial y que es en las zonas urbanas donde se presentan los más diversos procesos de interrelaciones económicas, sociales y políticas. En este sentido, La infraestructura cultural es definitiva para la conformación de identidades urbanas en el proyecto político de la Modernidad (Flores y Crawford, 2006). "Las ciudades son el marco idóneo en qué contemplar cómo se despliegan las nuevas formas de la identificación, destinadas a dar cuenta de la relación entre la sociedad y su entorno a través de una correspondencia simbólica" (Delgado, 1994:102).

La definición de la Identidad como concepto a partir del urbanismo, requiere de la interrelación de las diferentes definiciones de las ciencias humanas, ya que en la ciudad confluyen diferentes variables, que incluyen la dimensión espacial, geográfica, antropológica, sociológica y psicológica. En este sentido y teniendo en cuenta las diferentes definiciones expuestas previamente, la identidad urbana es una construcción colectiva a lo largo del tiempo y con repercusiones en el espacio; construcción que parte de las relaciones sociales sobre un territorio específico con características geográficas determinadas, dando lugar a diferentes evidencias espaciales que dan cuenta de una constante relación y dependencia entre el medio físico y las expresiones culturales, sociales y económicas, propias de un grupo humano. La ciudad como construcción temporal, representa la dimensión física de concreción de la cultura y la sociedad, conservando en sus diferentes espacios e intersticios, las huellas históricas de las relaciones humanas en su espacio. (Alva y Alderete, 2011). Entender la identidad como una categoría de análisis para el urbanismo, es esencial en la medida en que entrelaza la comprensión de su composición histórica y sus oportunidades futuras.

La ciudad se entiende a partir de diferentes componentes y sistemas, todos en continua relación e inherente correspondencia; en este orden de ideas, hablar de identidad desde el urbanismo, requiere identificar los diferentes componentes a partir de los cuales se construyen las huellas en el territorio y se explican las relaciones entre la sociedad y su entorno.

\subsubsection{Dimensión Natural}

El conjunto de circunstancias físicas, culturales, económicas y sociales que rodea a las personas y que actúa como condicionador de todos los signos individuales que se inscriben en el contexto, es lo que se denomina "medio ambiente" (Ullman, 2009). No obstante, La identidad Urbana desde la dimensión natural tiene en cuenta dos componentes en conjunción:

- La estructura de factores físicos derivados de la localización geográfica y los valores ambientales propios de una zona particular, se entiende también como contexto físico, es decir como un conjunto entrelazado de elementos dados en el espacio y el tiempo; esta dimensión puede ser abordada asimismo como paisaje, como sustento de vida y como territorio que permite establecer un sentido de pertenencia, aportando al desarrollo de una identidad cultural. 
- Relaciones sociales con el territorio, que dan cuenta de la relación entre los grupos humanos y su medio físico de localización.

La identidad urbana desde la dimensión natural, propone la comprensión de estos dos componentes relacionados, da cuenta de las condiciones particulares del contexto dadas por los elementos de la base natural, que incluyen al clima, el relieve, el suelo, la hidrología y la vegetación, extendiendo además su ámbito a la posibilidad productiva de la tierra incluyendo actividades pasadas y presentes que reflejan valores particulares del medio físico, traducidos en adaptaciones establecidas por los grupos humanos para el aprovechamiento de los diferentes recursos propios del territorio (FAO, 1976).

\subsubsection{Dimensión Socio cultural}

Para lograr que se establezca un vínculo identitario entre un individuo y el territorio que ocupa, es necesario que exista una "configuración significativa, de un conjunto de engranajes simbólicos que soporten y hagan practicable la identidad" (Delgado, 1994:102). En este orden de ideas, los Engranajes o puntos de contacto, juegan un papel fundamental en los procesos de construcción de identidades colectivas; se produce a partir de la relación social, un conjunto de significantes compartidos para la asimilación de símbolos comúnmente estáticos en el tiempo.

Referirse a la Identidad urbana desde su dimensión sociocultural hace referencia a la Memoria colectiva, un proceso de construcción social que se acumula a lo largo del tiempo y que se puede catalogar como construcción informal (Rosa, Bellelli y Bakhurst, 2000); lo anterior, significa que la memoria es una acumulación natural de experiencias y símbolos que son compartidos por una comunidad y que son transferidos a través del tiempo de forma simbólica, cultural y cotidiana. En este orden de ideas, la dimensión sociocultural, implica la valoración de las actividades cotidianas, las expresiones culturales y las tradiciones comunitarias relacionadas con los diferentes símbolos culturales adoptados por un grupo humano y los dispositivos de memoria espacial que albergan la significación entorno a diferentes actividades y/o períodos de tiempo representativos para una comunidad.

El componente sociocultural configura la dimensión intangible de la identidad en términos urbanos, en la medida en que reúne las representaciones estéticas de los diferentes individuos, sus costumbres, expresiones y manifestaciones que no pueden ser materializadas per se para su conservación, sino que requieren la transmisión cultural de los diferentes referentes simbólicos a través de los acuerdos colectivos de significación y los referentes físicos que se preservan en el tiempo como huellas materiales de las dinámicas intangibles.

Los sistemas sociales de significado que se encuentran relacionados directamente con la Identidad desde lo Sociocultural, pueden entenderse desde cuatro condiciones:

- Representan a la comunidad mediante la activación de memorias y recuerdos colectivos.

- Crean nuevas identidades culturales a partir de la transmisión generacional de costumbres.

- Poseen un carácter inductivo, es decir que conllevan a que la sociedad siga ciertas reglas, patrones y conductas.

- Evocan sentimientos específicos relacionados con la memoria colectiva (Rosa, Bellelli y Bakhurst, 2000).

Lo anterior permite ilustrar de forma más clara los aspectos de "lo urbano" que se asocian a la Identidad Sociocultural: El lenguaje, las expresiones folclóricas, las tradiciones orales, las expresiones musicales, la literatura, los mitos y productos de la oralidad, entre otras manifestaciones que subyacen en la cotidianidad de las ciudades y albergan en sí una relación entre la comunidad, su medio físico y su contexto histórico.

\subsubsection{Dimensión Histórico urbana}

Tal como se concibe desde otras disciplinas, la identidad Urbana es una construcción temporal en el espacio, que responde a diferentes condiciones contextuales a lo largo del tiempo. En este sentido, la identidad desde su dimensión Histórico-urbana, se concentra en los procesos históricos de mayor representatividad, que han dado lugar a transformaciones estructurales alrededor de la relación de la sociedad con su medio físico, configurando contextos particulares en los cuales se reconocen en los valores fundamentales de la conformación espacial de un asentamiento. 
La Dimensión Histórico Urbana, comprende dos aspectos fundamentales:

- El conocimiento de la historia urbana de un asentamiento particular, que permita establecer los hitos temporales claves para la comprensión de sus diferentes condicionantes contextuales, que han dado lugar a trasformaciones en los modos de vida, en la organización espacial, en las actividades cotidianas, en las relaciones territoriales con otros asentamientos, en el crecimiento urbano, etc.

- La identificación de las características físicas propias de los edificios representativos, la trama urbana, la organización de la ciudad, las actividades humanas, los referentes simbólicos y monumentales, el patrimonio material e inmaterial, y en general, todas las características que al ser reconocidas desde el presente, permiten referencias aspectos claves de la historia urbana de un asentamiento.

\subsection{Atributos Urbanos Identitarios como una propuesta metodológica de análisis}

La identidad urbana como categoría de análisis, permite realizar una lectura territorial que combina al espacio y al tiempo como factores dentro del proceso de construcción colectiva de los referentes simbólicos de una sociedad en torno a sus situaciones contextuales particulares. De igual forma, establece una relación entre las características actuales del territorio y su estructura de componentes naturales, artificiales y simbólicos, con los diferentes hechos históricos y factores ambientales que han conformado dichas particularidades.

Un atributo urbano identitario, es una expresión tangible de la relación indisociable de una sociedad con su entorno físico, que reúne características propias de su cultura, traduciéndose en cualidades diferenciadoras del territorio. Los Atributos urbanos identitarios, reúnen las tres dimensiones de la Identidad urbana: natural, sociocultural e histórico urbana; por lo tanto, se convierten en los contenedores de la información identitaria de un asentamiento, que más allá de ser características descriptivas, se convierten en el resultado de un análisis que permite una comprensión integral del territorio a partir del espacio y sus transformaciones, incluyendo la diversidad de variables que influyen en la construcción de la identidad y reconociendo sobre-todo, su carácter modificable, cambiante y en construcción permanente.

Realizar una lectura territorial con el fin de definir los atributos Urbanos identitarios propios de cada territorio, permite establecer las diferentes oportunidades que subyacen en los asentamientos para la consolidación de nuevas actividades, para las intervenciones proyectuales desde el punto de vista físico, identificar los diferentes ejes de comunicación con otros asentamientos susceptibles de ser fortalecidas y en general, posibilitan las intervenciones futuras a partir de las diferentes potencialidades que se han construido a lo largo del tiempo y que se mantienen en el espacio como configuración material de los diferentes procesos sociales y culturales.

Lo anterior, implica que los Atributos Urbanos son variables respecto a cada territorio y por lo tanto, el análisis asociado a los mismos como lectura territorial integral, requiere del establecimiento de unas categorías de análisis específicas, es decir, la delimitación de criterios constantes, para la definición de una identidad que es variable. En el siguiente apartado, se proponen los criterios para una lectura territorial que permitan la definición de los diferentes atributos urbanos identitarios, propios de un territorio, que posibilitan su lectura a partir de la identidad como categoría de análisis.

\section{CRITERIOS PARA LA LECTURA TERRITORIAL A PARTIR DE LA IDENTIDAD URBANA}

Los criterios para la lectura territorial a partir de la identidad urbana, son herramientas de análisis que permiten concluir en la determinación de los atributos urbanos de un asentamiento y por lo tanto, en sus cualidades identitarias; estos apuntan al análisis de las diferentes dimensiones de la identidad urbana: Natural, Socio-Cultural e Histórico Urbana; y se establecen como criterios constantes, para definir una identidad que es variable. En este orden de ideas, los criterios para la lectura territorial a partir de la identidad Urbana, se convierten en un sistema para el análisis y la lectura territorial, que al ser fijo puede aplicarse a diferentes territorios, para la definición de sus atributos urbanos particulares, cabe destacar que la base conceptual de la metodología propone un análisis a partir de la configuración histórica en tiempos y espacios de los asentamientos urbanos, bajo una lógica de palimpsesto urbano, que implica su aplicación a territorios consolidados en los cuales pueda hacerse una revisión 
profunda de su contexto histórico en relación con las condiciones actuales que se leen en la cotidianidad de los asentamientos.

Los Criterios para la lectura territorial a partir de la identidad, Tienen las siguientes características:

- Diversidad de contextos: Pueden ser aplicados en diferentes condiciones contextuales desde el punto de vista geográfico, es decir, puede aplicarse a territorios de diversa naturaleza (Valles, Corredores viales, Sistemas costeros, etc.), así como a cualquier zona independientemente de su localización.

- Diversidad de escalas: Pueden ser aplicados en diferentes escalas y ámbitos de análisis. Cabe aclarar que el resultado de los atributos identitarios como conclusión de la lectura territorial, tendrá una mayor definición y especificación en los territorios de menor escala, aunque a gran escala, sea posible la caracterización de grandes porciones de territorio a partir de atributos generales propios de la zona.

- Diversidad de Oportunidades: Teniendo en cuenta que apuntan a los diferentes componentes de la identidad urbana, la aplicación de los criterios deriva en atributos urbanos identitarios de diversa índole, los cuales permiten establecer diferentes oportunidades de intervención para un territorio específico en función de sus valores identitarios.

Además de lo anterior, es importante resaltar que reúnen tanto los diferentes factores del contexto histórico, como los múltiples condicionantes propios de su localización geográfica, ya que se requiere de estos dos aspectos para la definición de la identidad urbana de un territorio específico. Los criterios deben leerse desde la historia, desde la bibliografía relacionada con los estudios urbanos y territoriales de un asentamiento específico, desde el reconocimiento en campo de las diferentes características propias de una ciudad; y principalmente, a través de la cartografía como herramienta de espacialización de información recopilada a través de las diferentes fuentes.

La metodología propuesta, implica el reconocimiento de los diferentes criterios para la lectura territorial a partir de la identidad, a lo largo de los diferentes períodos de tiempo que configuran el contexto histórico de un asentamiento, permitiendo establecer la configuración Espacio-Temporal de los diferentes atributos urbanos identitarios propios del territorio. Esto quiere decir, que la aplicación de la metodología debe hacerse sistemáticamente a través de los períodos históricos que han conformado un territorio, evidenciando para cada uno de ellos, la aplicación de cada criterio.

\subsection{Definición de los Criterios para la lectura territorial a partir de la identidad urbana}

Los Criterios para la lectura territorial a partir de la identidad, parten de la dimensión espacial del territorio, entendiendo este como la base de configuración de las diferentes huellas históricas y el soporte de las diferentes relaciones sociales y humanas estructurantes de las condiciones actuales del asentamiento. No obstante el punto de partida es el espacio como objeto del análisis, transversalmente y de forma inherente, se abordan las diferentes relaciones sociales y características culturales de los grupos humanos, que hacen posible la conformación de la identidad urbana de un asentamiento y que son claves para explicar los diferentes hechos espaciales derivados de las adaptaciones humanas a un contexto ambiental, social y cultural determinado. La metodología de lectura territorial a partir de la identidad como categoría de análisis propone 5 criterios para ser abordados en la lectura, a partir de un análisis que relaciona tiempos y espacios a lo largo de la línea de tiempo que compone el contexto histórico de un asentamiento.

El análisis de los criterios para la lectura territorial a la luz de la identidad urbana, requiere combinar herramientas cartográficas y fotográficas que permitan establecer las relaciones de localización de las diferentes características del territorio así como sus diversas tipologías y formas en el mismo; para este fin y para lograr la aplicación de la metodología, se hace necesaria la revisión cartográfica y geográfica de los asentamientos a los cuales se aplique la lectura, teniendo en cuenta su posición geoestratégica, las relaciones con otros territorios, sus canales de comunicación aérea, vial y/o fluvial, entre otros aspectos propios de las relaciones territoriales a escala regional o nacional. Por otra parte, el trabajo de campo juega un papel fundamental en el proceso de análisis, ya que permite establecer una relación entre la identificación de los hechos espaciales fundamentales dentro del territorio y su localización cartográfica para el análisis de la composición urbana general de los atributos identitarios de un asentamiento. 
El trabajo de campo implica necesariamente la relación con los diferentes actores que protagonizan las diferentes relaciones sociales en el territorio, por tanto, para la recopilación de la información se hace de gran importancia la vinculación de los aportes de diferentes líderes sociales, sobre todo aquellos que han tenido una directa relación con la configuración del territorio a lo largo de la historia, que dan cuenta de la composición de las economías locales (Artesanos, pescadores, agricultores, etc.; según sea el caso); protagonistas de los hechos culturales trascendentes al espacio (líderes de juntas culturales locales, artistas independientes, entre otros), así como los diferentes personajes que se encuentran en constante relación con las dinámicas ambientales y la explotación de los recursos de base, entre los que caben líderes ambientales, promotores culturales, entre otros. Para la vinculación de estos actores, se proponen a partir de la metodología dos posibilidades: el acompañamiento en los diferentes recorridos que se hagan necesarios en el trabajo de campo y la elaboración de cartografías participativas en el reconocimiento de los diferentes hechos espaciales característicos de territorio.

\subsubsection{Estructura Natural como Ordenadora del Territorio.}

Este criterio hace referencia a los componentes que estructuran la condición natural del territorio, topografía, hidrografía, condiciones ambientales, clima, etc. La lectura territorial de estos estructurantes, requiere poner en relación las pre-existencias, con las consecuencias espaciales que parten de la relación entre un grupo social y su entorno. Este criterio, se relaciona directamente con la definición de la identidad desde el punto de vista ambiental y del paisaje, por lo cual, lo más importante resultan ser las consecuencias de las modificaciones que realiza el hombre en su contexto natural y viceversa; pero también, permite establecer aspectos claves de las características naturales propias de su geolocalización. Este criterio propone el reconocimiento de:

- Condiciones propias de la base natural que se establecen como características principales de composición del paisaje.

- Actividades humanas que obedecen al aprovechamiento de los recursos aportados por el entorno físico.

- Huellas de la relación entre la sociedad y el medio ambiente, que pueden ser desde las oportunidades que ofrece o desde las diferentes amenazas que representa.

- Condiciones del orden urbano o formas de ocupación del espacio relacionadas con estructurantes de orden natural.

El análisis de este criterio, permite identificar atributos urbanos relacionados con las posibilidades que ofrece el medio físico, con los lineamientos para el asentamiento y crecimiento urbano en función de los diferentes componentes de la estructura natural, las adaptaciones propias de las comunidades para mejorar el aprovechamiento de los recursos ofrecidos por el medio ambiente y los diferentes sistemas que se desarrollan desde la economía, la infraestructura y la configuración urbana, para hacer frente a los retos propuestos por la estructura natural pre-existente.

\subsubsection{Orden Primario de Conformación Espacial para la comprensión de la configuración Urbana actual.}

Este criterio hace alusión a la forma como se reflejan en la composición de la forma urbana de la ciudad, las diferentes condiciones contextuales que han influído en un asentamiento desde el punto de vista económico, cultural y social, para la configuración de características morfológicas, jerarquías urbanas y órdenes del espacio físico. Se orienta al reconocimiento de:

- Los hechos generadores de la ciudad, es decir, las actividades, hechos o condiciones que propiciaron la consolidación del asentamiento como nodo urbano.

- La estructura urbana espacial de su origen, reconocer la condiciones morfológicas que dieron origen a la expansión del asentamiento; es decir, si hace parte de un trazado ortogonal, a un desarrollo espontáneo, lineal, en nodos, etc.

- Las condiciones contextuales que condicionaron la estructura urbana, los diferentes aspectos económicos, sociales o de infraestructura que influyeron en la organización morfológica de la ciudad y su crecimiento en el tiempo 
- Las problemáticas y oportunidades actuales que se presentan partir de la configuración morfológica del asentamiento, que obedecen a diferentes aspectos históricos que han marcado el crecimiento urbano.

El análisis de este criterio permite establecer relaciones comparativas y asociativas entre la conformación original de la ciudad desde el espacio y las condiciones actuales de su morfología y organización urbana. En este orden de ideas, permite establecer criterios de intervención para potencializar las oportunidades que devienen de la morfología urbana y la adaptación al medio físico, o por el contrario, definir los elementos claves a tratar en un proceso de renovación de las condiciones particulares del orden urbano, para contrarrestar las problemáticas identificadas.

\subsubsection{Vocación Territorial Como posibilidad de diversificación económica.}

La vocación territorial es una característica comúnmente asociada con el concepto de identidad urbana, no obstante, es solo un componente de la misma, el cual relaciona al espacio con las diferentes actividades productivas de una zona específica. El reconocimiento de la vocación territorial, implica conocer las diferentes actividades productivas representativas a las cuales se ha dedicado la población de un asentamiento a lo largo del tiempo. Este criterio propone abordar los siguientes factores en el proceso de lectura territorial:

- Actividades económicas que dieron origen al asentamiento urbano o que potencializaron su desarrollo.

- Actividades económicas que generaron transformaciones de la infraestructura vial o de transporte, en función de la producción, comercialización o distribución de uno o varios productos.

- Servicios y actividades que hayan marcado las actividades laborales de la sociedad perteneciente al asentamiento y a su vez, hayan atraído población flotante o permanente generando crecimiento urbano.

- Actividades económicas que modifican la configuración espacial particular de una zona o de la totalidad del asentamiento, reflejándose en la morfología y en la tipología edificatoria.

- Actividades del orden productivo que hicieron parte de la historia y han desaparecido, las que permanecen y las actividades emergentes que proponen cambios estructurantes en el asentamiento desde el punto de vista espacial y social.

Este criterio permite identificar oportunidades de diversificación económica, que propicien el fortalecimiento social a partir de la inserción de nuevas actividades, la potencialidad de actividades económicas históricas, la puesta en valor de la infraestructura o los productos de diferentes períodos del desarrollo de la ciudad, la reconfiguración de acciones sociales y la planificación urbana, para potencializar las actividades emergentes y fortalecer los renglones que han caracterizado al asentamiento a lo largo de la historia.

\subsubsection{Conexiones Urbanas para el Fortalecimiento Territorial.}

Las conexiones urbanas y la relación territorial con otros asentamientos, es clave para la conformación de la identidad urbana. Por lo anterior, este criterio propone abordar las diferentes conexiones físicas, culturales y económicas, entre un asentamiento y otros nodos urbanos estratégicos que han propiciado su desarrollo, se benefician directamente de sus productos o que guardan una relación histórica desde el punto de vista cultural. Las conexiones urbanas se leen y se evidencian en:

- Vías estratégicas de comunicación y conexión con otros asentamientos que son claves para explicar su crecimiento urbano y su desarrollo económico.

- Caminos históricos de relación con otros grupos sociales y otros territorios.

- Sistemas de transportes no vehiculares que establecen relaciones con otros asentamientos.

El reconocimiento de estas relaciones y sus canales de comunicación, permite establecer las debilidades con las que cuenta el sistema territorial de conexiones propio de un asentamiento particular y a su vez, las oportunidades de conexión que son claves para fortalecer las relaciones con otros asentamientos a partir de las intervenciones estratégicas desde la infraestructura y/o la logística de transporte.

\subsubsection{Diversidad Tipológica Urbano Arquitectónica para su aprovechamiento.}


Este criterio, hace referencia al reconocimiento de los referentes simbólicos de orden espacial que se conforman a partir de la arquitectura, el espacio público y la morfología urbana, las huellas históricas de diferentes actividades que se hayan dado en el territorio y que se mantienen en formas espaciales a las cuales se les han dado usos diferentes o se conservan sin uso específico. La diversidad tipológica urbana Arquitectónica se reconoce a través de:

- Tipologías de espacio público asociadas a actividades específicas de la economía o la cultura.

- Arquitectura industrial, comercial o de servicio propia de actividades productivas antiguas.

- Arquitectura doméstica característica, que obedece a condiciones climáticas, de composición familiar e incluso, de características productivas.

Este criterio es clave para abordar temáticas relacionadas con la identidad desde el punto de vista sociológico e incluso psicológico, teniendo en cuenta que el reconocimiento de estas tipologías, es clave para la valoración de los diferentes espacios que conforman las huellas históricas de un asentamiento, en la medida en que permiten identificar diferentes estrategias técnicas desarrolladas a lo largo del tiempo para dar frente a diferentes condiciones del contexto ambiental; permiten reconocer los valores estéticos de referenciación que dan cuenta de conexiones culturales y tradiciones sociales, apuntan al reconocimiento de los referentes físicos de identificación social y posibilitan el aprovechamiento de los diferentes espacios a partir de sus valores tipológicos específicos.

\section{CONCLUSIONES}

Comprender los fenómenos urbanos emergentes presentes en los asentamientos urbanos contemporáneos, implica necesariamente el reconocimiento de los diferentes factores contextuales que conforman la historia de la ciudad, las características de su contexto geográfico y los condicionantes externos de sus dinámicas propias. Reconocer la ciudad como una construcción en tiempos y espacios, es clave para una lectura territorial que de manera integral, combine los diferentes factores que desde la dimensión natural, social e histórico-urbana, han dado lugar a las condiciones existentes en los asentamientos y por lo tanto, sus oportunidades futuras.

La identidad es un concepto ampliamente abordado desde diferentes disciplinas, pero desde el punto de vista del urbanismo requirió una construcción a partir de múltiples visiones, que posibilitaran una integralidad para la comprensión del concepto, teniendo en cuenta que los componentes de la ciudad son múltiples, que sus actores son sociales y que su contenedor es un espacio físico.

La lectura territorial a partir de la identidad, se propone como una categoría de análisis para el reconocimiento de oportunidades de intervención en los procesos de planeación y ordenamiento a cualquier escala. Además de los tres componentes fundamentales (Natural, Sociocultural, Histórico Urbano), la lectura territorial a la luz de la identidad implica el reconocimiento de criterios de análisis a partir de los cuales se puedan comprender las particularidades que identifican a cada asentamiento. Dichos criterios se propusieron como un sistema fijo aplicable en diferentes escalas y diferentes contextos, aportando así conclusiones variables a partir de las características particulares de cada territorio estudiado, definiendo sus atributos urbanos característicos y estableciendo un punto de partida para la determinación de lineamientos de intervención a partir del reconocimiento de los valores fundamentales propios del territorio que se reflejan en la construcción de su identidad.

En este orden de ideas, la identidad se convierte en una forma de reconocer las características propias de un territorio, que aunque son muy particulares, son cambiantes, modificables, y en constante transformación y construcción, por lo anterior, la pregunta por la identidad, no solo se responde a partir del contexto histórico, sino también de las relaciones geográficas.

Para efectos de la investigación que soporta este artículo, los atributos identitarios se reconocen a través del espacio como dimensión tangible de la ciudad, pero la comprensión de la identidad urbana implica interrelacionar procesos sociales que configuran la importancia del espacio en la construcción del territorio y características propias de las actividades humanas que configuran las estructuras de relación entre la dimensión social y el espacio construido; es por esto que para el reconocimiento de la identidad territorial, se hace necesario: 
- La investigación histórica que permita establecer los diferentes períodos y hechos que determinan las características propias del contexto.

- El análisis cartográfico que posibilita la identificación de los principales estructurantes urbanos, la composición del espacio urbano y la configuración morfológica de los asentamientos.

- El trabajo en campo que permite identificar las actividades propias de la población, el acercamiento a las dinámicas cotidianas que se entretejen en el espacio físico, las condiciones del entorno natural y en términos generales, la interrelación de las variables que determinan los procesos urbanos que definen la identidad urbana de un asentamiento.

Es por lo anterior, que se considera como una oportunidad dentro de los procesos de lectura territorial previo a los hechos de proyectación urbana, teniendo en cuenta que los atributos identitarios componen los puntos clave de las relaciones sociales y humanas con el territorio y por tanto, representan las mayores oportunidades para la transformación del territorio, en la medida en que a partir de los atributos identitarios es posible identificar los diferentes factores contextuales que respaldan los fenómenos urbanos actuales que se encuentran en los asentamientos consolidados. Esta propuesta metodológica, representa un instrumento replicable de lectura territorial, que posibilita la consideración de diferentes variables, incluyendo la histórica, para determinar las características particulares de un asentamiento, para plantear criterios de intervención en función de mejorar y/o potencializar sus principales valores urbanos y principalmente, para el reconocimiento de las diferentes oportunidades con las que cuenta un territorio en función de maximizar su importancia territorial a partir de sus valores locales.

\section{BIBLIOGRAFÍA}

ALVA,

FLORES, P y CRAWFORD, L. (2006). Identidades sin espacios de memoria. El caso B y ALDERETE, L. (2011). Identidad Urbana, Reflexiones sobre las orientaciones teóricas para su estudio. Teuken Bidikay, Revista Latinoamericana de investigación en organizaciones, Ambiente y Sociedad, 2, 213-229.

CASTELLS, M. (1998). La Era de la Información, El poder de la Identidad. Buenos Aires: Siglo XXI Editores.

DELGADO, M. (1994). Las Estrategias de Memoria y Olvido en la construcción de la identidad urbana: El Caso de Barcelona. VII Congreso de la antropología en Colombia, Medellín, 15-18 de junio.

del área metropolitana de barranquilla (Colombia). Investigación \& Desarrollo, 14, 352-371.

FOOD AND AGRICULTURE ORGAN.ZATION -FAO- (1976). A Framework for land evaluatión. FAO Soils bulletin, 32, 1-87.

HALL, S y DU GAY, P. (2011) Cuestiones de Identidad Cultural. Madrid: Amorrortu.

HARNER, J. (2001). Place identity and copper mining in Sonora, Mexico. Annals of the Association of American Geographers, 91, 660-680.

PORTAL, M. (1991) Diversas perspectivas en la construcción teórica de la identidad: una bibliografía básica. Alteridades, 1 122-125.

ROCA, Z. (2012). Territorial Identity and Regional Development. Planning The IDENTERRA Model. International Seminar on Strategic Planning Methods of Analysis of the Potential for Territorial Development, San Petesburgo, 10-19 de junio.

ROSA, A.; BELLELLI, G. y BAKHURST, D.; (2000) Memoria Colectiva e Identidad nacional. Madrid. Biblioteca Nueva, 2000.

SASSEN, S. (2007) El reposicionamiento de las ciudades y regiones urbanas en una economía global: ampliando las opciones de políticas y gobernanza. Revista EURE, 33, 100, 9-34

\section{FUENTES ELECTRÓNICAS}

https://redalebrije.wordpress.com (Consulta 14/08/2016). 